\title{
ALICE's adventures in cultural computing
}

\section{Jun $\mathrm{Hu}^{*}$, Christoph Bartneck, Ben Salem and Matthias Rauterberg}

\author{
Department of Industrial Design, \\ Eindhoven University of Technology, \\ PO Box 513 \\ 5600 MB Eindhoven, The Netherlands \\ E-mail: j.hu@tue.nl \\ E-mail: c.bartneck@tue.nl \\ E-mail: b.i.salem@tue.nl \\ E-mail: g.w.m.rauterberg@tue.nl \\ *Corresponding author
}

\begin{abstract}
In the paradigm of cultural computing, different cultures need different approaches to address the cultural determinants that strongly influences our way of thinking, feeling and worldview in general. For the western culture, our answer to this need is an artistic and interactive installation (ALICE) based on the narrative 'Alice's Adventures in Wonderland'. To address the western culture characteristics highlighted in the narrative, six stages were selected and implemented as an interactive experience. From start to end, the user undergoes an immersive environment that integrates embodied and virtual agents, real and nature mimicking, and both virtual and augmented reality. Every stage challenges the hardware and software design to provide the intended experience, which at the overall system level yet have to be seamlessly integrated. A distributed and multi-layered architecture is designed to accommodate this need. After several pilot tests, the installation is ready as a cultural computing platform for the experiments that address the western cultural determinants.
\end{abstract}

Keywords: alice's adventures in wonderland; culture; cultural computing; human-computer interaction.

Reference to this paper should be made as follows: Hu, J., Bartneck, C., Salem, B. and Rauterberg, M. (2008) 'ALICE's adventures in cultural computing', Int. J. Arts and Technology, Vol. 1, No. 1, pp.102-118.

Biographical notes: Jun $\mathrm{Hu}$ is an Assistant Professor in the Designed Intelligence Group at the Department of Industrial Design, Eindhoven University of Technology, The Netherlands. His background is in Mathematics (BSc), Computer Science (MEng), User-System Interaction (PDEng) and Distributed Software Architecture (PhD). He is also a qualified system analyst and senior programmer.

Christoph Bartneck is an Assistant Professor in the Department of Industrial Design at the Eindhoven University of Technology. He has a background in Industrial Design and Human-Computer Interaction, and his projects and studies have been published in various journals, newspapers and conferences. His interests lie in the fields of social robotics, design science, and multimedia applications. He has worked for several companies including the Technology Centre of Hannover (Germany), LEGO (Denmark), Eagle River Interactive (The USA), Philips Research (The Netherlands) and ATR (Japan). 
BEn Salem is an Assistant Professor in the Department of Industrial Design at the Eindhoven University of Technology. He has a background in Architecture (Master's) and Electronics (PhD). In his work, he would like to exploit his combined expertise in the area of interactive devices, virtual environments, user interfaces and robotics.

G.W. Matthias Rauterberg received a BS in Psychology (1978) at the University of Marburg (Germany), a BA in Philosophy (1981) and a BS in Computer Science (1983), an MS in Psychology (1981) and an MS in Computer Science (1986) at the University of Hamburg (Germany), and a PhD in Computer Science/ Mathematics (1995) at the University of Zurich (Switzerland). Since 1998, he is a Full Time Professor for 'Human Communication Technology' first at IPO, Center for User System Interaction Research and later at the Department of Industrial Design at the Eindhoven University of Technology (TU/e, The Netherlands). From 1999 to 2001, he was a Director of IPO. He is now the Head of the Designed Intelligence research group at the Department of Industrial Design of the TU/e.

\section{Introduction}

Over last few decades, the paradigm of human-computer interaction has evolved from personal computing towards cooperative and social paradigms. The computer is no more centre of interest, nor is it the focus of the attention of the user. It is the benefits and effects on the user that matter. Along this line of evolvement, a new paradigm is proposed to address the cultural determinants (attitudes, norms, values, beliefs, actions, communications and groups, etc.) that have since ancient times, a strong influence on our ontology and epistemology, and the influence of computing on these cultural determinants and vice versa (Rauterberg, 2006a,b,c). This new paradigm is based on Kansei-Mediated Interaction (Nakatsu, Rauterberg and Salem, 2006). Kansei Mediation is a form of multimedia communication that carries non-verbal, emotional and Kansei information (e.g. unconscious communication). The main research objectives in KanseiMediated Interaction are underlying almost unconscious cultural determinants (see also $\mathrm{Hu}$ and Bartneck, 2005; Salem and Rauterberg, 2005a,b). In this context, cultural computing is more than integrating cultural aspects into interaction. It is about allowing the user to experience an interaction that is closely related to the core aspects of his or her culture (the cultural determinants). As such, it is important to understand one's cultural determinants and how to render them through the interaction.

These cultural determinants distinguish a culture from the others. Over last 3,000 years of civilization, these determinants formed four distinct groups in which people created distinct religious and philosophical traditions: Confucianism and Daoism in China; Hinduism and Buddhism in India; monotheism in middle east; and philosophical rationalism in Greece. To study the cultural determinants, we have investigated illustrative stories that are well known, classical, accessible, and also relevant from the cultural computing point of view. We primarily looked for narratives that would be helpful in the understanding of the determinants of both the Eastern and the Western cultures. To this effect, we have selected the story of 'Zen Buddhism' attributed to Boddidharma (circa $500 \mathrm{AC}$ ), and 'Alice's Adventures in Wonderland' by Carroll (1865). Both are examples either to help understand the underlying cultural value (i.e. Zen) or question it (i.e. Alice). For the Eastern and Western culture, the main value dealt with is enlightenment, but in different ways. Utilising on modern technology, 
Nakatsu, Rauterberg and Salem (2006) try to give a new direction in form of 'Kansei Mediation' to enable societies transforming towards enlightenment (also see Rauterberg, 2004). Salem and Rauterberg (2005a,b) discuss the relationship of cultural computing and entertainment, and Hu and Bartneck (2005) could conclude that 'culture matters'.

In the East, the concept enlightenment is regarded as a state of perfect freedom in which one is awakened to the eternal and ultimate truth that is the reality of all things. With the spread of Zen Buddhism in the 13th century, the religion cultivated self-discipline and austerity as the path to enlightenment. Meditation is at the centre of Zen practice and many Zen art forms can be seen as vehicles for inward reflection or as visualisations of the sudden and spontaneous nature of enlightenment. Sansui (landscape, literally 'mountain and water') ink painting is one of these Zen art forms. Executed with black ink on white rice paper using bamboo brushes, Sansui painting communicates beauty and emotion through simple and pure means, yet leaves spaces for imagination and contemplation. Tosa, Matsuoka and Thomas (2004) and Tosa et al. (2005) projected this style of communication into an Eastern sansui world: the ZENetic Computer. The ZENetic computer is an interactive system with which the user can create a virtual world by manipulating 3D images of sansui paintings. Based on the user's sansui design, the system infers his or her internal consciousness and generates a story that the user can 'enter' and that further shakes the user's consciousness. ZENetic Computer was and still is an ambitious project that tries to deliver the essence of an ancient Eastern culture by means of Western technology to create an interactive experience dealing with complex issues such as human consciousness and unconsciousness.

In the West, the enlightenment is a 'way out' process that releases us from the statues of 'immaturity' - a certain state of our will of accepting someone else's authority in areas where the use of reason is called for (Kant, 1784). Nisbett et al. (2001) can confirm that Westerners are analytic whereas the Easterners are more holistic. The Westerners pay attention primarily to the object and use rues, including formal logic, to understand its behavior. To address logic reasoning in the western culture the most appealing narrative is 'Alice's adventure in Wonderland' of Carroll (1865). His facility at word play, logic and fantasy has delighted audiences ranging from the most naïve to the most sophisticated. The book has remained popular since its publication and has influenced not only generations of children, but also a number of major 20th century writers such as James Joyce and Jorge Luis Borges.

Not only the logic in reasoning, but also the other Western cultural determinants are highlighted in 'Alice's Adventures in Wonderland', such as absolute truth and certainty, self-reflection, Cartesian logic in space, linearity in time, and many others. From the perspectives of both its popularity and its cultural coverage, the book 'Alice's Adventures in Wonderland' can serve as input for a Cultural Computing project in the West.

Inspired by the above thoughts, we started the cultural computing project ALICE, aiming at an interactive system that is equivalent to ZENetic computer in the East. Our approach is to create an artistic and interactive experience based on the cultural determinants as highlighted in the story 'Alice's adventures in Wonderland'. Alice's adventures are illustrative of English culture. Indeed, English and Western culture in general are based on Monotheist religions (Judaism, Christianity and Islam) which are concerned with certainty and absolutism in the sense of absolute truth and certainty. Western culture is also based on Cartesian logic and a linear and constant flow of time. In the story 'Alice's adventures in Wonderland', Alice, who is tired of the rational world, she lives in and therefore follows the white rabbit into a hole, leading to a world without 
rational boundaries, experiences several culture shocks in this new world. Alice adventures happen in a world of paradox, the absurd and the improbable. The key aspects of Alice in Wonderland can be resumed in the following points:

1 a non-linear and non-constant time flow

2 a distortion of experience in space and with other characters

3 a counter-intuitive, common sense defying heuristics.

To be able to investigate the effects of cultural computing on the user's experience, we have built an interactive installation with six selected stages of experiences, in line with the 'Alice's adventures in Wonderland' plot (albeit shortened). Each stage is aiming at a particular aspect of the user experience (Table 1). Next, these six stages are described in detail, after which the system implementation of the installation is briefly described. The measurements are discussed thereafter.

\section{Alice's adventures in six Stages}

The ALICE installation was build inside of the W-Hal building of the Eindhoven University of Technology (Figure 1). It takes a space of $12 \mathrm{~m}$ by $12 \mathrm{~m}$ spanning two floors over a total height of $7 \mathrm{~m}$.

Table 1 Alice's adventures in six stages

\begin{tabular}{lllll}
\hline Stage & Theme & Experience & Measurements \\
\hline 1 & In the park & Time & Boredom and curiosity & $\begin{array}{l}\text { Retrospective duration } \\
\text { Presence }\end{array}$ \\
2 & Down the rabbit hole & $\begin{array}{l}\text { Environmental } \\
\text { space }\end{array}$ & Disorientation \\
3 & Eat Me and Drink Me & $\begin{array}{l}\text { Personal space } \\
\text { Genesis }\end{array}$ & $\begin{array}{l}\text { Shrinking and growing } \\
\text { Swimming, out of water }\end{array}$ & $\begin{array}{l}\text { Presence } \\
\text { Qhe pool of tears }\end{array}$ \\
$\begin{array}{l}\text { Advice from a } \\
\text { caterpillar }\end{array}$ & Self & $\begin{array}{l}\text { Questioning the concept of } \\
\text { self }\end{array}$ & $\begin{array}{l}\text { Personality, self- } \\
\text { esteem and -concept }\end{array}$ \\
6 & The Cheshire cat & Logic & $\begin{array}{l}\text { Challenged by the logic } \\
\text { reasoning }\end{array}$ & Generating alternatives \\
\hline
\end{tabular}

Figure 1 The Alice installation inside the W-hal building (see online version for colours)

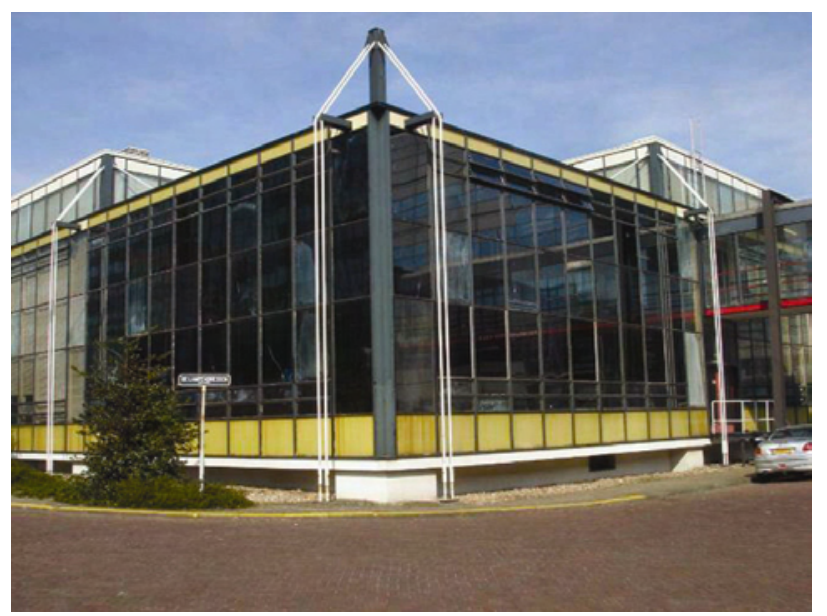


Figure 2 Schematic drawing of the Alice installation

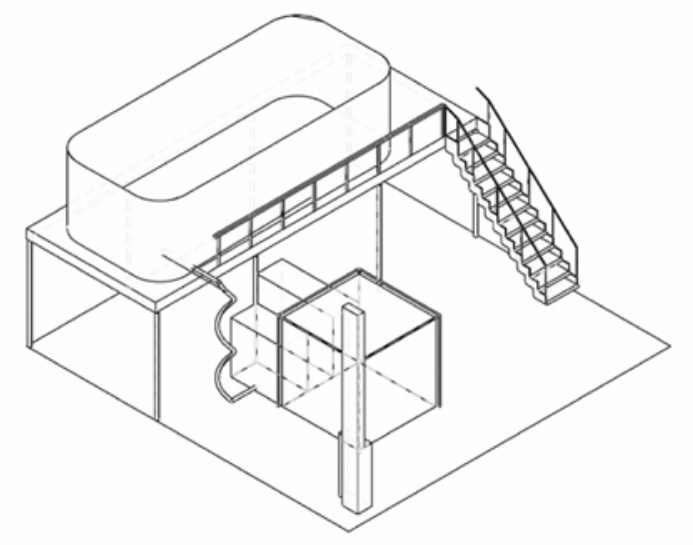

Figure 3 A visitor follows the rabbit (see online version for colours)

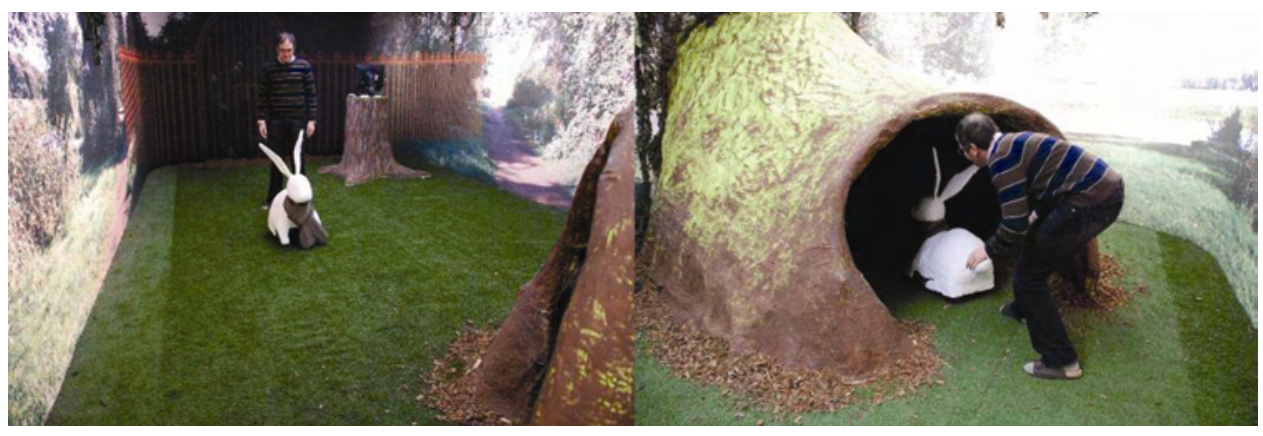

The ALICE Installation consists of six consecutive stages. The visitor walks by $\mathrm{him} /$ herself through them, one after the other. Given the Western Culture's emphasis on individuality, the installation is designed for individual experiences. Figure 2 shows a schematic drawing of the installation. Notice the panoramic room on top, the spiral rail for the electric seat and the Cave in front. We will explain the function of each part in more detail within each of the corresponding stages. For each stage, the corresponding section of Carroll's original story is firstly summarised, followed by an impression of the design and the implementation.

\subsection{Stage 1: in the park}

In the first scene of Carroll's original book, Alice is bored and sleepy sitting on a bank with her sister. A white rabbit runs close by her and while looking at its pocket watch it cries out 'Oh dear! Oh Dear! I shall be late!'

Stage 1 symbolises the concept of time. The visitor of the ALICE Installation enters this stage through two curtains. Inside, a $360^{\circ}$ panorama picture of Cambridge surrounds the visitor, the place where Carroll lived and worked. The panorama depicts a location in nature at the river Cam (Figure 3). 
Figure 4 A visitor descents through the rabbit hole (see online version for colours)

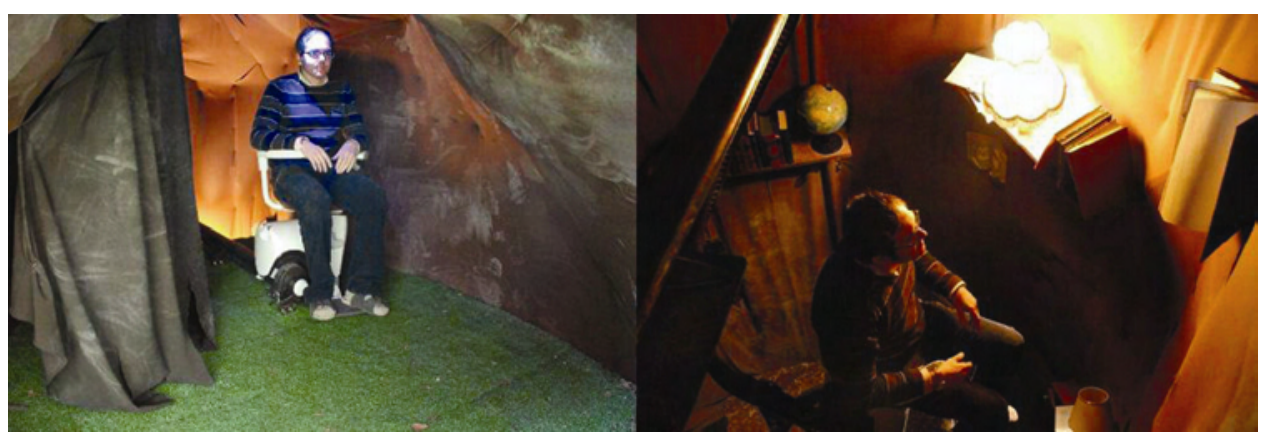

The visitor is told to first fill in a questionnaire at a computer that is located on top of a tree trunk. Afterwards, the visitor is left to his/her own devices. The visitor has to wait until he/she is bored and thereby enters a similar state of mind as Alice in the original study. Then, a white rabbit appears from behind the curtain. It has a pocket watch and says through its internal speakers 'Oh dear! Oh Dear! I shall be late!' before disappearing into a rabbit hole at the far end of the room. On its way pass the visitor it tries to seduce the visitor to follow by starting a catch-me-if-you-can type of behavior. The rabbit enters a hidden section inside of the rabbit hole before the visitor enters the hole and hence disappears from sight. The visitor enters the rabbit hole after the rabbit and thereby enters stage 2 .

The panorama picture was printed on a large fabric. Several lights behind the fabric lighten the room and thereby giving the panorama the brightness of a summer day. The rabbit was build using a carbon fiber fabric. The model is mounted on top of a remote controlled car. A wide-angle video camera mounted in the ceiling allows the experimenter to see the whole room and thereby enable him to efficiently control the rabbit with the remote control.

\subsection{Stage 2: down the rabbit hole}

Alice falls down the rabbit hole in an unusual fashion. The speed of her fall is very slow and she is able to interact with objects that are attached to the walls of the rabbit hole, such as cupboards and bookshelves. The fall takes such a long time that Alice engages in a conversation with herself before dozing off. At last, she lands on a heap of sticks and dry leaves. Stage 2 can be characterised by environmental space. Alice is discussing whether she can fall right through the earth and come out in New Zealand. She disputes the relationship of herself within the space of the earth.

When entering the rabbit hole in the ALICE installation, the visitor finds an electric seat that is mounted on a rail. Once safely seated, the seat takes the visitor down a tunnel in spiral movement (Figure 4). Along the walls of the tunnel, cupboards, bookshelves and lamps are mounted. The speed of the electric seat is slow.

The seat was build using an electric stair lift, commonly used in the houses of elderly to enable them to move from one floor to another. A securely controlled gate prevents the visitor to pass by the seat and thereby endangering him/her to truly fall down the rabbit hole. The seat is only activated once the armrests are put down, which prevents the visitor from falling off while moving. Infrared cameras are placed inside the rabbit hole to allow 
the experimenter to monitor the visitor. Since both, stages 3 and 4 are associated with the concept of space, we will discuss the possible measurements together in Section 2.3.

\subsection{Stage 3: Eat Me and Drink Me}

Alice enters a dark corridor with many doors, which are all locked. She approaches a glass table on which a small golden key lays. She uses the key to open a tiny door that leads to a garden, but Alice is too tall to enter. She approaches the table again, and this time she notices a bottle labelled 'Drink Me' and later a little cake labelled 'Eat Me'. By drinking from the bottle, she shrinks and by eating the cake, she grows. Eventually, she manages to have the appropriate size to enter through the tiny door.

Similar to stage 2, stage 3 is associated with the concept of space. Clearly, we could not grow or shrink the visitors directly, but we were able to manipulate their relative size in comparison to the environment by using a cubic CAVE. The walls and the ceiling were made of white semi-transparent material. We used the back-projection method to project a seamless virtual environment onto the walls of the CAVE. Figure 5(a-c) shows a scale model of the specially designed CAVE. A sliding side is connected to the entrance and the exit tunnels, enabling a 5-side full projection when the visitor is in the CAVE. Both the entrance and the exit tunnels are also build as a 3-side projection CAVE, but for the time being only the exit tunnel is used for the stage 4 (the pool of tears).

Figure 5 Scale modle of the CAVE (a-c) and the virtual room (d) (see online version for colours)

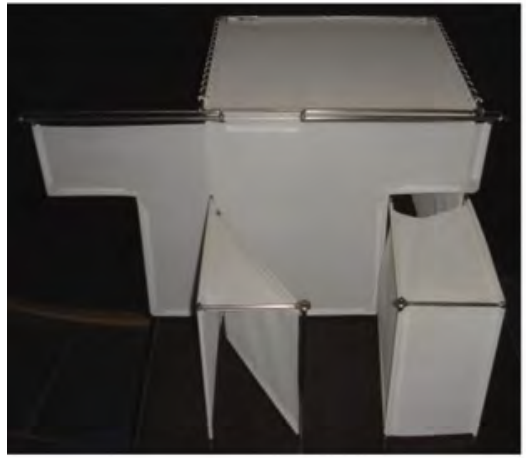

(a) entrance open

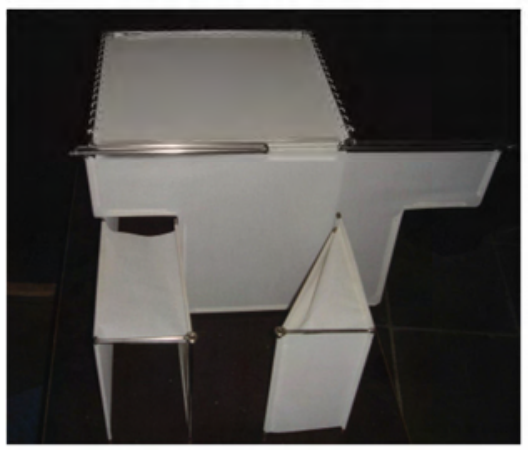

(c) exit open

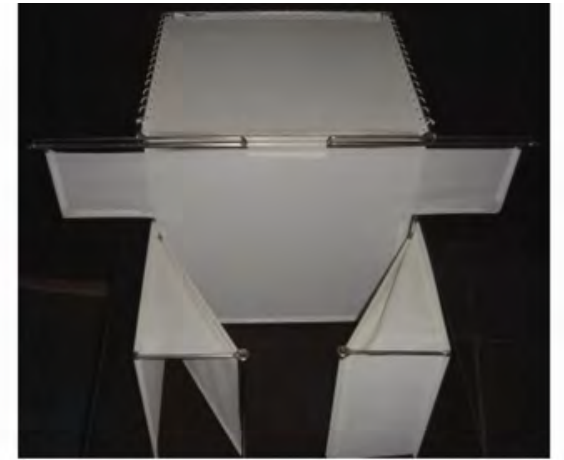

(b) both entrance and exit closed

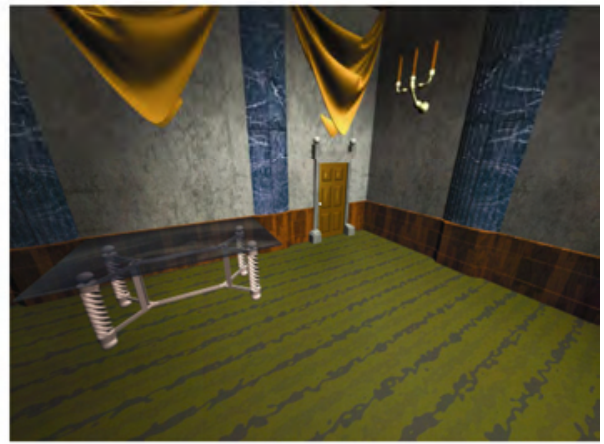

(d) Virtual room 
Figure 6 Eat Me and Drink Me (left), visitor eating the cookie (right) (see online version for colours)

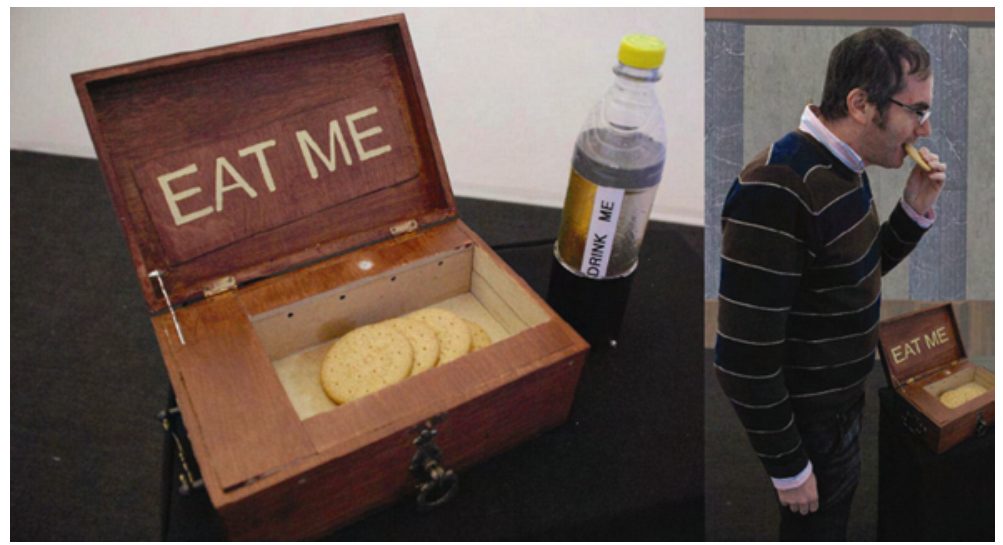

The visitor entered the cave and had the impression to stand in a virtual room (Figure 5(d)). A cookies box labelled 'Eat Me' and a bottle labelled 'Drink Me' are placed on top of a small table (see Figure 6). When the visitor drinks from the bottle, the virtual room enlarges, giving the impression that the visitor is shrinking. When eating the cookie, the virtual room shrinks, giving the visitor the impression that he/she is growing. On one side of the room, a door is shown. Once the visitor reaches the appropriate size, the wall on which the door is shown moves aside and thereby allows the visitor to enter stage 4.

We used the Vizard VR Toolkit from Worldviz to synchronise the five projectors. The model of the virtual room was developed in 3D Studio Max. The floor of the cave is equipped with pressure sensors that allow us to determine the visitor's position in the Cave. Depending upon his/her location, the perspective of the projection is adjusted to give a true 3D impression of the virtual room. The bottle features touch and tilt sensors to detect drinking. The cookie box is equipped with a microphone that allows us to detect the visitor's chewing sounds when eating the cookie.

\subsection{Stage 4: the pool of tears}

During her growing and shrinking experience, Alice cries many tears. When she walks through the tiny door she enters a pool of tears. She talks to a mouse that swims along side her and together with some other animal they finally reach the shore. When the visitor enters stage 4, the sea with a mouse in it is projected on one of the walls (see Figure 7). In addition, a smoke machine creates an impression of moisture and adds a mystical feeling to this stage. The visitor walks along the projection and thereby enters stage 5 .

At the beginning, we were discussing building a real pool, but it was not clear what the visitors would do with their clothes. Hence, we created a virtual pool of tears with the use of back-projection. The animation of the mouse in the water was created in 3D Studio Max. 
Figure 7 Pool of tears (see online version for colours)

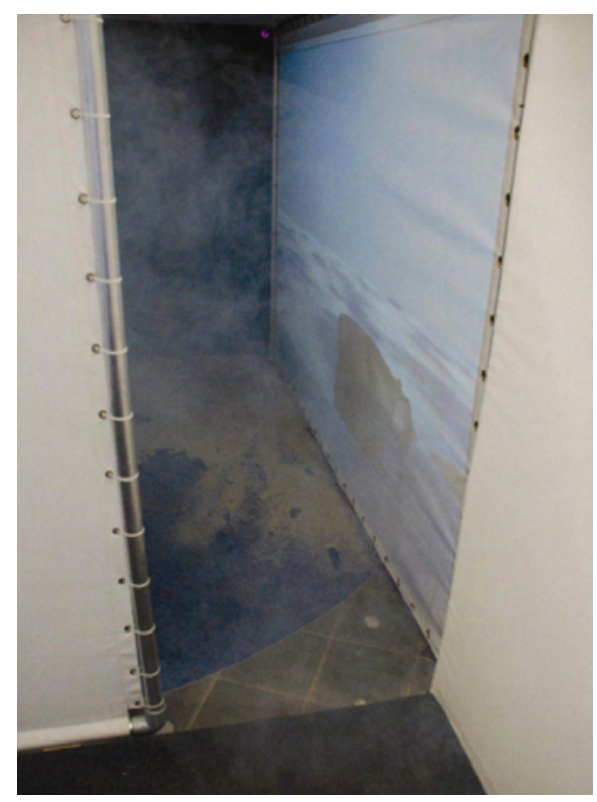

Figure 8 A visitor talking to the caterpillar (see online version for colours)

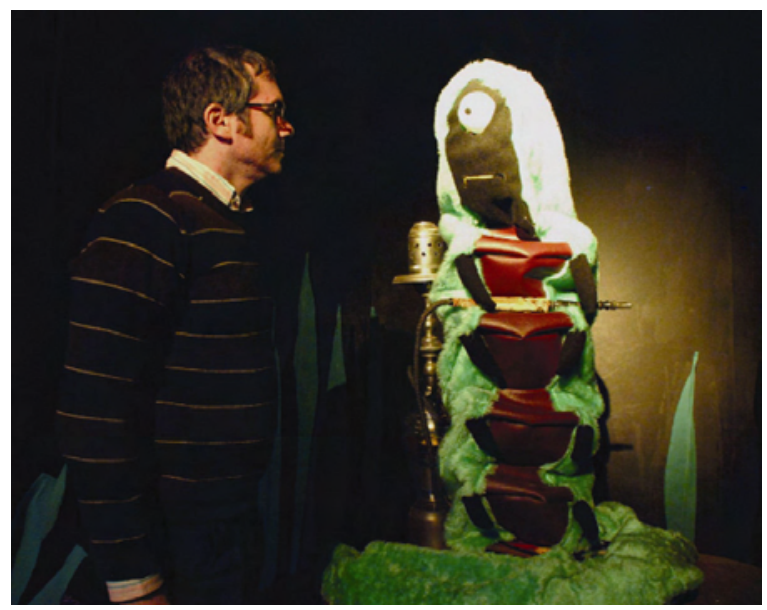

\subsection{Stage 5: advice from a caterpillar}

The caterpillar engages Alice in discussion about herself. It asks Alice who she really is and how she wants to be. The complete episode is a dialogue and hence we created a robotic caterpillar to engage the visitor in a similar dialogue (see Figure 8). Microphones recorded the utterances of the visitor and a simple dialogue system manages the conversation. Since most of the questions are metaphysical or mystical, a conclusive dialogue can be created without an extensive Artificial Intelligence (AI) for the caterpillar. 
This stage can be associated with the concept of the self. Throughout history, there have been wide varieties of theories about the self, coming from the fields of philosophy, psychology, and religion. This includes assertions that there is no self; that the idea is a logical, psychological or grammatical fiction; that the sense of self is properly understood and defined in terms of brain processes; that it is merely a constructed sociological locus, or the centre of personal and public narratives, or that it belongs in an ineffable category on its own (Gallagher and Shear, 2000). There is a significant difference in the construction of the self when comparing European/American culture and the Japanese culture (Kitayama et al., 1997; Nisbett and Masuda, 2003). By addressing the Western individual self-concept, Alice's self is challenged in 'Advice from a Caterpillar'. After she entered the rabbit hole to follow the White Rabbit, she experienced a lot of transformations both physically and mentally. This brought her in an initial state of confusion, which is emphasised in her conversation with the Caterpillar: 'Who are YOU?' This challenging attitude of the caterpillar makes Alice uncertain about herself, becoming vulnerable and open for persuasion (Fogg, 2003). Such a situation gives the possibility for a confrontation with and stimulates awareness of the self-concept. The character symbolised as a caterpillar is well chosen. One of the most important characteristics of caterpillars and butterflies is their unique life cycle. One of nature's most mysterious metamorphoses occurs when a caterpillar changes from a slow-moving, fat and ugly creature to a colorfully winged, beautiful butterfly. This metamorphosis happens to a lot of insects, but not as dramatically as it does to a butterfly (Heiligman, 1996). In this respect, the 'caterpillar' character can unconsciously pursue the message to a human user not to be afraid of a fundamental metamorphosis in his or her self-concept. This symbolic meaning can counterbalances the challenges intended by a conscious dialog next to it.

\subsection{Stage 6: the Cheshire cat}

The Cheshire cat involves Alice in a dialogue about logical reasoning and madness. During the dialogue the cat disappears at times completely and sometimes only its grin remains visible. This episode of Carroll's book is predominantly a dialogue. In contrast to the previous stage, we did not use a robotic character, since the transformations (Figure 9) of the cat are impossible in reality.

Figure 9 Transformations of the Cheshire cat (see online version for colours)

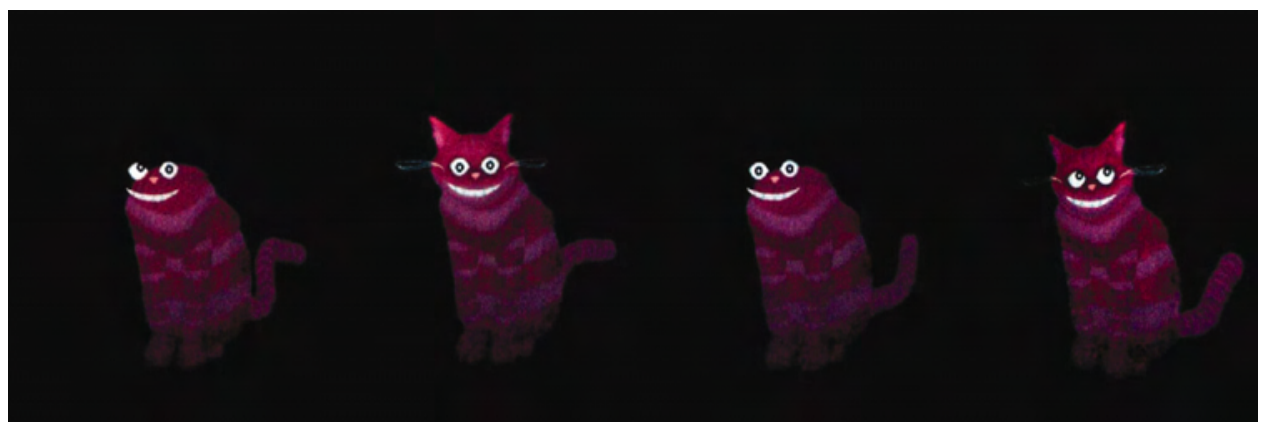


Figure 10 A visitor talks to the Cheshire cat (see online version for colours)

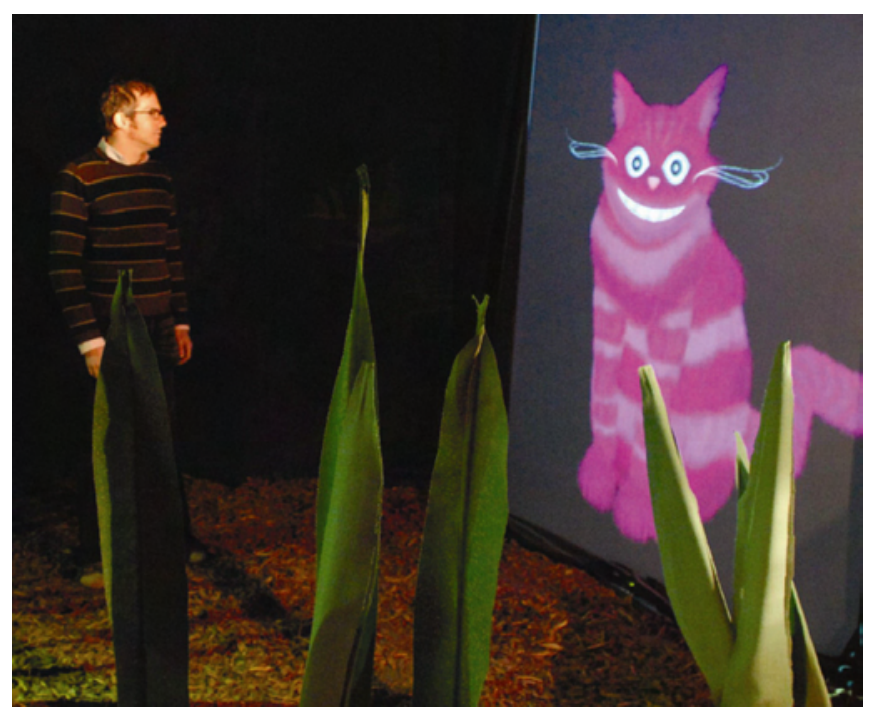

Therefore, we created a virtual Cheshire cat that is projected on a screen (see Figure 10). When the visitor approaches the cat, it engages him/her in dialogue similar to the one in Carroll's book.

A Microphone was hidden next to the screen to record the visitor's responses. A simple 2D animation tool (Adobe Director) is used for the animation of the cat and the dialogue management. Similar to the Caterpillar, most of the questions are metaphysical or mystical, and hence a conclusive dialogue can be created without an extensive Artificial Intelligence (AI).

\section{System architecture}

In the Alice installation, every stage has its own requirements on the setup and employs a different set of technology - from animated virtual characters to physical robots; from CAVE-based virtual reality to augmented virtual reality; from still image prints to simulated real environments. Every stage uses one or more computers, connecting to different type of microcontrollers or hardware interfaces that are again connected to a variety of sensors and actuators. It is a challenge of its own how these software and hardware bits and pieces can be structured together into one automated system that brings the visitor a smooth and holistic experience.

A multi-layered, -agent based structure is designed based on the distributed architecture proposed by $\mathrm{Hu}$ (2006), in which Presentation-Abstraction-Control (PAC) agents (Coutaz, 1987, 1997) are distributed over a network and connected through so-called Channels (see Figure 11). In this architecture, a channel generalise the data and control communication among agents. In Figure 11, the channels are depicted by a straight line connecting a data supplier $(\bullet)$ and a data consumer $(\circ)$. The agents at every level have actuators ( $\square$ ) presenting data to the environment and sensors ( $\square$ ) detecting the input from the environment. 
Figure 11 Distributed Presentation-Abstraction-Control over channel connections

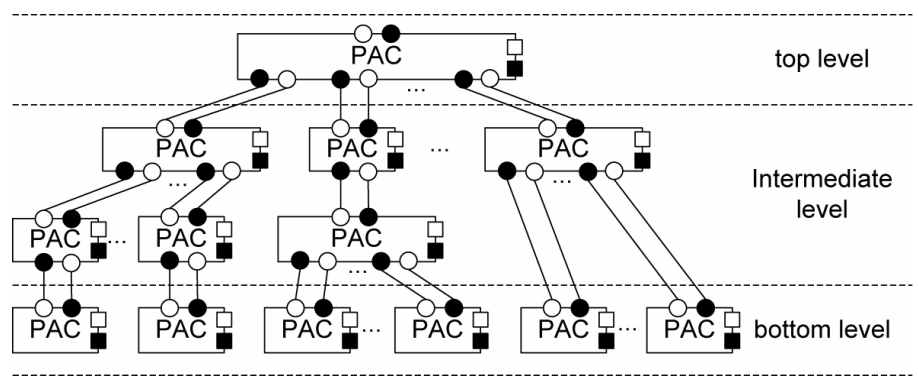

Figure 12 Artistic and Interactive Installation architecture

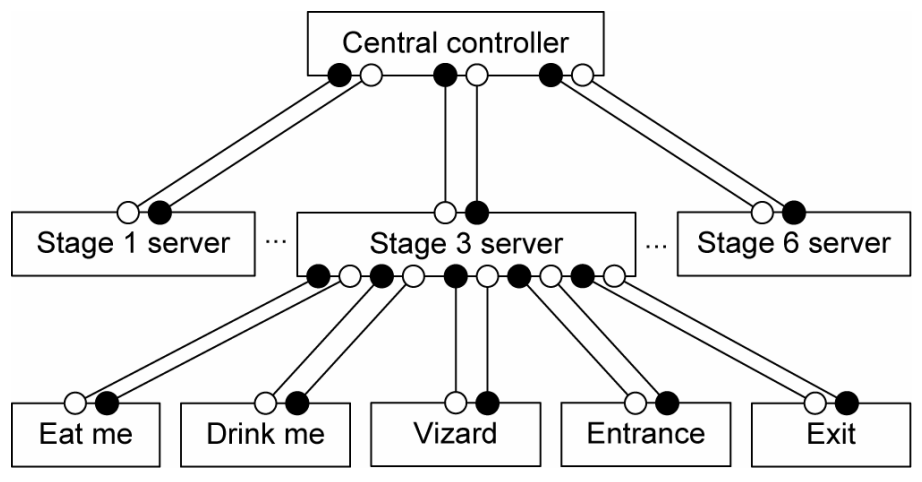

Applying this structure to the Alice installation results in a three-layer structure depicted in Figure 12. At the top level is the central controller that coordinates the stages based on a scripted scenario. Every stage implements a stage server that updates the central controller with the local states and interaction events and receives the commands from the central controller. At the bottom level are software components (e.g. Vizard in Figure 12), or programmable hardware interfaces that are directly connected to sensors and actuators. The stage servers coordinate their bottom level agents and hide the local complexity from the top level controller.

This structure enables the ALICE project to decompose the complexity of the entire installation into loosely coupled stages, and to organise the project in a similar hierarchical manner that distributes the tasks in smaller teams and coordinates the progress at a higher level.

\subsection{XML-based messages through channels}

The channels between the bottom level and the intermediate level are mostly implemented over Universal Serial Bus (USB) or serial cable connections or over Bluetooth radio. The channels between the central controller and the stage servers are implemented over Transmission Control Protocol (TCP)/Internet Protocol (IP) network connections. There are well established technologies and protocols that could have been used for these channel connections, for example Remote Procedure Call Protocol (Srinivasan, 1995), Simple Object Access Protocol (Gudgin et al., 2003) and Common 
Object Request Broker Architecture (CORBA) (Bolton, 2001). However, they require the specific software infrastructure to be implemented and supported by all the involved parties. We were not able to find one that supports completely Java, Flash, Python and Max/MSP that are used for different stages. Hence, an XML-based communication protocol is designed and implemented directly on top of TCP/ IP, to ensure the minimum requirement on the software packages or programming languages used by the stage servers. All messages comply the following form:

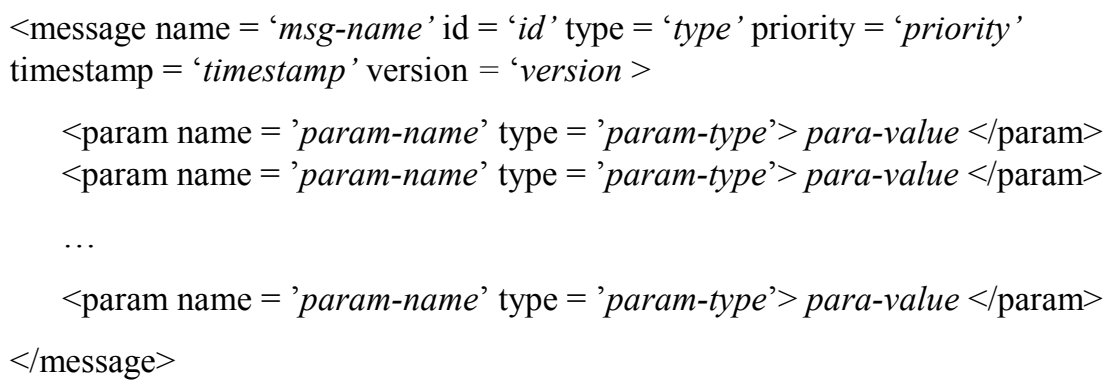

\subsection{Stage state transitions}

To connect all the stages seamlessly and also to ensure the minimum requirements on the implementation of the channel messages, a stage is required to implement the following four stages, the stage transition commands from the central controller, and the stage change notifications to the central controller:

\section{prepared|opened|closed|stopped}

When a stage is prepared, everything should ready so that the stage can be opened for a user to enter the stage. When the user leaves the stage, the stage should then be closed. At any time, the stage can be stopped because of emergency for example - which means all the ongoing activities are stopped immediately. A stage can be cleared and reset at any time to the initial state (prepared) so that it can be opened again for the user to enter. A limited number of commands are required to be implemented by the stage servers for the state transitions as shown in Figure 13.

Figure 13 Stage stage transitions (see online version for colours)

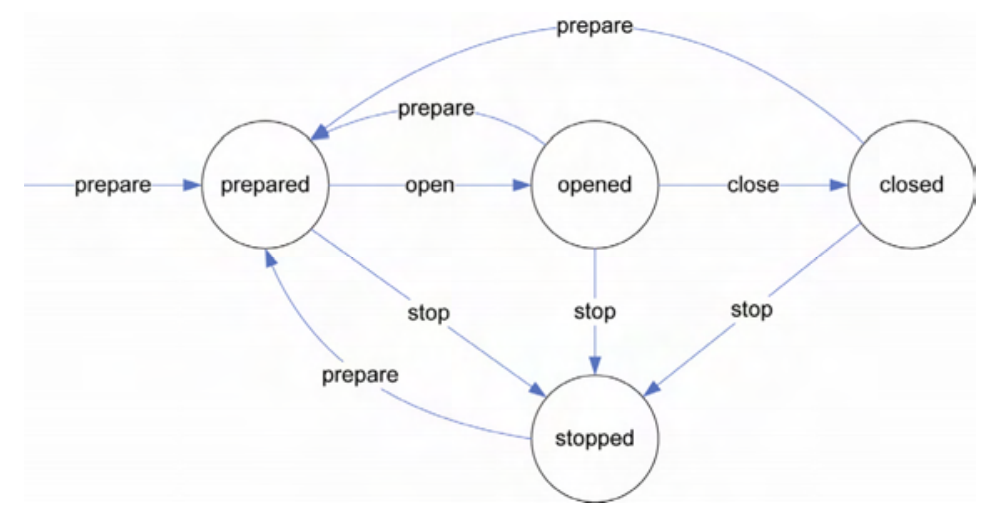




\section{Experiences and measurements}

It is likely that the Alice installation will have an effect on its visitors, but it is unclear what effects and how strong they may be. The visitors' experience may range from being mildly entertained to a deeper psychological disturbance. Currently, we are planning a first experiment and in the following paragraphs we describe the approach we intend to take. The experiment will be setup as a repeated measure study. Each participant will be measured before and after visiting the Alice Installation. The within-subject setup will decrease the effect of individual differences.

Since the effect of the Alice installation is unknown, we intend to use several measurement instruments that cover a wide range of concepts. This wide spread of measurements will reduce the risk that we may miss an important effect. In a second experiment, we will then be able to focus on those measurement instruments that proofed to be sufficiently reliable and sensitive. We will discuss our choices for measurement instruments in order of the six stages in the Alice installation and their corresponding theme.

After two preliminary studies of single stages (Kooijmans and Rauterberg, 2006), we are currently planning a first experiment for the complete installation. The experiment will be setup as a repeated measure study. Each participant will be measured before and after visiting the Alice Installation. The within-subject setup will decrease the effect of individual differences.

The first stage is associated with the concept of time. Fraisse (1984), Nakatsu, Rauterberg and Salem (2006) argued that

"the more one pays attention to time, the longer it seems, with the extreme being expectancy which is nothing but expectancy of a desired or feared event. Reciprocally, duration seems short when the task is difficult and/or interesting."

Since, we are not primarily interested in the influence of expectation on the perception of time (e.g. 'A watched kettle never boils' (Flaherty, 1999)) we will focus on retrospective duration similar to Glicksohn's (1992) study. His study showed that retrospective estimation of the duration to an altered sensory environment was not affected by the environment, but the method of production registered an influence on the participants' cognitive timer.

Stages 2 and 3 deal with space. Stage 2 can be characterised by environmental space, while stage 3 is associated with personal space. In both stages, the relationship between the visitors' and the installation's space is of essence. This relationship can be described within the well-established framework of Presence. The Independent Television Commission - Sense of Presence Inventory (ITC-SOPI) questionnaire (Lessiter et al., 2001) is the de facto standard tool to measure presence and has previously demonstrated its usefulness (Hu and Bartneck, 2005). We therefore included it in our study.

The symbolic interpretation of the stage 4 remains difficult. A possible symbolism could be the Christian ritual of baptizing. We were not yet able to identify an operationalisation of the genesis concept for this stage. This stage is also very short transitional phase in the installation and hence we may neglect. We will continue our effort and would be grateful for suggested from the reader.

Stage 5 is associated with the concept of the self. The Alice installation is expected to not only influence the consciousness, but also the unconsciousness of its visitors. We intend to measure the visitors' conscious perception of the self through a personality test 
based on the NEO Five-Factor Inventory (NEO-FFI) with 60 items, which is designed to take only $15 \mathrm{~min}$ to fill in. For the unconscious self-concept Greenwald, McGhee and Schwartz (1998) developed the Implicit Association Test to overcome this limitation and demonstrate its effectiveness to measure the self-esteem and -concept (Greenwald and Farnham, 2000).

The necessity of logical reasoning is the topic of the last stage. Generating alternatives is a key component in human reasoning (Newstead, Thompson and Handley, 2002) and hence we use the Alternative Generation Task originally proposed by Torrens, Thompson and Cramer (1999). Participants are being asked to create as many diagrams as they could think of to visualise a multiple model syllogism. Their responses were scored in terms of the number of different but correct diagrams produced.

\section{Conclusions}

We have introduced and discussed an artistic and interactive installation that is used as a research platform for the new field named cultural computing, based on six particular parts in the narrative 'Alice in Wonderland' (Carroll, 1865) that are designed to deal with specific cultural determinants, such as space, time, self and. We described the six stages of our installation, taking into account the corresponding part in Carroll's original book, the translation into the visitor experience inside the installation, a short description of the technologies used. A distributed and multi-layered architecture is designed to deal with the challenges brought up by different hardware and software infrastructures used in different stages. Proposals are also made for measuring the effects of each stage on the visitors. After several pilot test we were able to conduct two preliminary studies (Kooijmans and Rauterberg, 2006) which indicated the great potential of the installation.

Overall, we believe that the ALICE installation can be seen as valuable contribution to cultural computing by providing access to the unconscious determinates of the Western culture. The effect of the installation on the visitors by using a mixture of conscious and subconscious instruments is yet to be measured. This will then reveal the full potential of Alice's wonderful story which remained in the public attention for already over 140 years.

\section{Acknowledgements}

This project is sponsored by Microsoft Research in Cambridge, UK. We want to thank Sjriek Alers, Chet Bangaru, Geert van den Boomen, Dirk van de Mortel, Peter Peters, Christoph Seyfert for their valuable contributions to our project.

\section{References}

Bolton, F. (2001) Pure Common Object Request Broker Architecture (CORBA). Indianapolis, IN: Sams.

Carroll, L. (1865) Alice's Adventures in Wonderland. London, UK: Macmillan.

Coutaz, J. (1987) 'PAC, an implemention model for dialog design', Paper presented in the Proceedings of the Interact'87, Stuttgart, September, pp.431-436.

Coutaz, J. (1997) 'PAC-ing the architecture of your user interface', Paper presented in the Proceedings of the 4th Eurographics Workshop on Design, Specication and Verication of Interactive Systems (DSV-IS'97) (pp.15-32). Heidelberg, New York, NY: Springer. 
Flaherty, M.G. (1999) A Watched Pot: How We Experience Time. New York, NY: New York University Press.

Fogg, B.J. (2003) Persuasive Technology: Using Computers to Change What We Think and Do. Amsterdam, The Netherlands; Boston, MA: Morgan Kaufmann Publishers.

Fraisse, P. (1984) 'Perception and estimation of time', Annual Review of Psychology, Vol. 35, pp.1-36.

Gallagher, S. and Shear, J. (Eds) (2000) Models of the Self. Exeter, UK: Imprint Academic.

Glicksohn, J. (1992) 'Subjective time estimation in altered sensory environments', Environment and Behavior, Vol. 24, pp.634-652.

Greenwald, A.G. and Farnham, S.D. (2000) 'Using the implicit association test to measure self-esteem and self-concept', Journal of Personality and Social Psychology, Vol. 79, pp.1022-1038.

Greenwald, A.G., McGhee, D.E. and Schwartz, J.K.L. (1998) 'Measuring individual differences in implicit cognition: the implicit association test', Journal of Personality and Social Psychology, Vol. 74, pp.1464-1480.

Gudgin, M., Hadley, M., Mendelsohn, N., Moreau, J-J. and Nielsen, H.F. (2003) 'Simple object access protocol (SOAP) Version 1.2 specification', W3C Recommendation.

Heiligman, D. (1996) From Caterpillar to Butterfly. New York, NY: Harper Collins.

Hu, J. (2006) Design of a Distributed Architecture for Enriching Media Experience in Home Theaters, Department of Industrial Design. Eindhoven, Eindhoven University of Technology. PhD.

Hu, J. and Bartneck, C. (2005) 'Culture matters - a study on presence in an interactive movie', Paper presented in the Proceedings of the 8th Annual International Workshop on Presence, London, pp.153-159.

Kant, I. (1784) 'Beantwortung der Frage: was ist Aufklärung?' Berlinische Monatschrift, Vol. 2: pp.481-494.

Kitayama, S., Markus, H.R., Matsumoto H. and Norasakkunkit V. (1997) 'Individual and collective processes in the construction of the self: self-enhancement in the United States and self-criticism in Japan', Journal of Personality and Social Psychology, Vol. 72, pp.1245-1267.

Kooijmans, T. and Rauterberg, G.W.M. (2006) 'Advice from a Caterpillar: an application for cultural computing about the self', Supplement of 5th International Conference on Entertainment Computing (ICEC) (pp.5-8), Sanda.

Lessiter, J., Freeman, J., Keogh, E. and Davidoff, J. (2001) 'A cross-media presence questionnaire: the ITC sense of presence inventory', Presence: Teleoperators and Virtual Environments, Vol. 10, pp.282-297.

Nakatsu, R., Rauterberg, M. and Salem, B. (2006) 'Forms and theories of communication: from multimedia to Kansei Mediation', Multimedia Systems, Vol. 11, pp.304-312.

Newstead, S.E., Thompson, V.A. and Handley, S.J. (2002) 'Generating alternatives: a key component in human reasoning?', Memory and Cognition, Vol. 30, pp.129-137.

Nisbett, R.E. and Masuda, T. (2003) 'Culture and point of view', Paper presented in the Proceedings of the National Academy of Sciences, Vol. 10019, pp.11163-11170.

Nisbett, R.E., Peng, K., Choi, I. and Norenzayan, A. (2001) 'Culture and systems of thought: holistic versus analytic cognition', Psychological Review, Vol. 108, pp.291-310.

Rauterberg, M. (2004) 'Positive effects of entertainment technology on human behavior', in R. Jacquart (Ed.), Building the Information Society, IFIP (pp.51-58). Dordrecht, The Netherlands: Kluwer Academic Press.

Rauterberg, M. (2006a) 'From personal to cultural computing: how to assess a cultural experience', in G. Kempter and P. von Hellberg (Eds), uDayIV - Information nutzbar machen (pp.13-21). Lengerich, Germany: Pabst Science Publisher. 
Rauterberg, M. (2006b) 'How to assess the user's experience in cultural computing', in T. Bosenick, M. Hassenzahl, M. Müller-Prove and M. Peissner (Eds), Usability Professionals 2006 (pp.12-17). Nobelstraße, Stuttgart: Fraunhofer Informationszentrum Raum und Bau.

Rauterberg, M. (2006c) 'Usability in the future - explicit and implicit effects in cultural computing', in A.M. Heinecke and H. Paul (Eds), Mensch \& Computer 2006: Mensch und Computer im StrukturWandel (pp.29-36). München, Germany: Oldenbourg Verlag.

Salem, B. and Rauterberg, M. (2005a) 'Aesthetics as a key dimension for designing ubiquitous entertainment systems', in M. Minoh and N. Tosa (Eds), 2nd International Workshop on Ubiquitous Home - ubiquitous society and entertainment (pp.85-94). NICT Keihanna and Kyoto.

Salem, B. and Rauterberg, M. (2005b) 'Power, death and love: a trilogy for entertainment', Lecture Notes in Computer Science, Vol. 3711, pp.279-290.

Srinivasan, R. (1995) 'RPC: remote procedure call protocol specification Version 2', RFC 1831, Sun Microsystems, Inc. ONC Technologies.

Torrens, D., Thompson, V.A. and Cramer, K.M. (1999) 'Individual differences and the belief bias effect: mental models, logical necessity, and abstract reasoning', Thinking and Reasoning, Vol. 5, pp.1-28.

Tosa, N., Matsuoka, S. and Thomas, H. (2004) 'Inter-culture computing: ZENetic computer', Paper presented in the Proceedings of the International Conference on Computer Graphics and Interactive Techniques: ACM SIGGRAPH 2004 Emerging Technologies (p.11). Los Angeles, CA: ACM, doi: http://doi.acm.org/10.1145/1186155.1186167, isbn: 1-59593-896-2.

Tosa, N., Matsuoka, S., Ellis, B., Ueda, H. and Nakatsu, R. (2005) 'Cultural computing with context aware application: ZENetic computer', Lecture Notes in Computer Science, Vol. 3711, pp.13-23. 